\title{
University Students Use of Explanatory Models for Explaining Electric Current in Transitory Situations
}

\author{
Ane Leniz, Kristina Zuza, Jenaro Guisasola* \\ Department of Applied Physics, University of the Basque Country (UPV/EHU), Polytechnical College, Spain
}

Copyright (C) 2015 by authors, all rights reserved. Authors agree that this article remains permanently open access under the terms of the Creative Commons Attribution License 4.0 International License

\begin{abstract}
There are many studies on students' understanding of DC circuits in the steady state, but few studies have been made about students' ideas on transient states of movement of charges in a conductor. The traditional Electricity curriculum often involves situations of transient motion of charges such as the process of charging a body (conductor or dielectric), closing or opening the switch in a DC circuit or, circuits charging and discharging capacitors. In this research, we present two questions that have been used to investigate the representations of students about the movement of charges of transients in direct current, which focus on the transition between electrostatics and electrodynamics in first year university undergraduate study. The results obtained show that a significant percentage of students cannot correctly interpret simple transitory state current phenomena. Their explanations fall into two general categories. Firstly, one based on potential difference and secondly, one that excludes current flow in processes of transitory state. Some consequences for teaching are discussed.
\end{abstract}

Keywords Physics Education, University Students' Difficulties on Learning Explicative Models, DC Simple Circuit in Transitory State

\section{Introduction}

In many introductory physics courses on electricity, the core of the theory of electric circuits is a set of simple DC circuit laws, which relate algebraically voltages, currents and resistance. These laws are included because they are usually related to Drude's classical theory of electric conduction. The Drude model gives an adequate classical description of electric conduction in metals which leads to Ohm's law and shows that resistivity in a metal can be explained by the motion of its free electrons [1]. One might assume that all details about the simple system of DC circuit are well known and understood. However, in a DC circuit with resistors, explanations to predict relationships between variables I, V and $\mathrm{R}$ refer, in general, only to the steady state, and express invariance with respect to certain transformations. These relationships do not describe how circuits behaves during transient states, and therefore, provide little understanding of the underlying dynamic processes that lead to the equilibrium state of a system [2]. Moreover, the traditional electricity curriculum at introductory physics courses often involves situations of transient motion of charges, such as the processes of charging a body (conductor or dielectric), closing or opening the switch in a DC circuit or, circuits charging and discharging capacitors.

Understanding students' ideas about how DC circuits work in physics is one important aspect of providing guided instruction and of curriculum development in electricity. There are many studies that identify which concepts and representations students learn well and with which of them students struggle within the context of DC circuits in the steady state, but few studies have been made of students' ideas on transient states of movement of charges in a conductor $[3,4]$. In this research, we present two questions that have been used to investigate the representations of students about the movement of charges of transients in direct current, which focus on the transition between electrostatics and electrodynamics in first year at university.

One major goal of our work was the development of a guided problem-solving (GPS) tutorial [5] to be used in an introductory electricity course to help students connect the explanatory model of direct current in simple circuits with the concepts of electrical field and potential as defined by electric theory. However, this paper mainly focuses on the students' difficulties that we have identified as a result of addressing the following research questions:

- Which type of ideas or models do students use when explaining transient state current in a conductor wire?

- To what extent do students use the Drude model to explain the movement of charges?

We will present the development of instructional strategies used to address these questions in a separate paper.

\section{Context of the Research and Methodology}

The current research involved more than 70 students at the 
University of the Basque Country (UPV/EHU) in the first year Introductory Physics course for engineers. All of them had taken at least two years of physics in high school and had passed the national exams in Spain for admission to University to study science or engineering. Those students received 3.5 hours of lectures and 2 hours of laboratory per week during 14 weeks (second semester) on electromagnetism. Electrostatics and electric circuits were taught for 5 or 6 weeks of this course.

In addition, the study also involved more than 200 students from the University of Washington (UW) in the calculus-based E\&M course for scientists and engineers. The weekly format of this course is three lectures of 50 minutes each, one two-hour laboratory and one tutorial section based on Tutorials in Introductory Physics [6] of 50 minutes in small groups.

In all cases, lectures were given by experienced teachers of the Physics Department, and the Electricity curriculum in both universities is similar to those given in textbooks like Tipler and Mosca (2004).

The data were collected in written questions, some of which were online. All the questions were answered as post-test after receiving instruction. The students' answers to the questions were subjected to rigorous analysis [7]. The analysis does not focus on correct or incorrect answers but on identifying students' understanding and alternative conceptions. We are aiming at a nuanced understanding of what aspects of explanatory model on currents transient state students understand reasonably well and what aspects are problematic for them. One member of the research team derived a draft set of categories of description for each question based on a reading of the students' answers, and tentatively allocated each answer to one of the draft categories. Three weeks later, the same researcher again read the students' answers and repeated the process. The intra-rater reliability Cohen's kappa coefficient was calculated for this period of time, obtaining a mean value of 0.89 for all the questions, which is satisfactory for a confidence level of $95 \%$. The other researchers carried out the task of allocating each answer to one of established categories independently. Once the answers were classified, the allocations of answers were compared. The mean kappa reliability coefficient was 0.86 for the questions, which indicates very good consistency in the judges' criteria for setting the categories described.

\section{Experimental Design and Results}

Two questions were given to students over the course to investigate their understanding on the mechanism of how current works in transitory movement of charges. The questions were administered in different formats, but all of them were done post-test after receiving instruction. One of the questions was a multiple-choice question which also asked for a reasoned explanation of the answer. The second question was an open-ended question in which students were asked to explain in depth the phenomenon presented. Although the students' curriculum at both universities is similar, it is not the aim of this study to make comparisons or rankings. What this study seeks to identify is the students' main thinking patterns when interpreting contexts of current in transitory state and whether or not there are similarities in the responses collected in the different countries. The questions and a few examples are discussed below.

The first question aims to study what students think about the relations between current and potential difference in a DC circuit (figure 1). The question Q1 is very familiar to students in the academic context.

Question Q1.- In the circuit shown below, what is the potential difference between point $\mathrm{A}$ and point $\mathrm{B}$ after the switch is closed? Choose the correct option (the resistance of the wire is ignored)
a) $0 \mathrm{~V}$
b) $+6 \mathrm{~V}$.
c) Infinity
d) $+12 \mathrm{~V}$.
e) Not enough information to answer

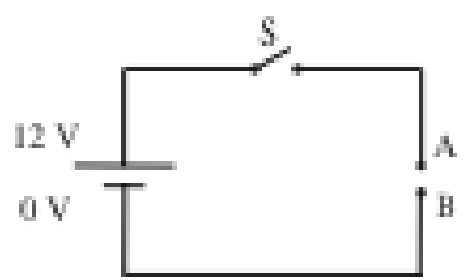

Figure 1. A battery and wires make up the circuit. The resistance of the wires is considered zero. At the UPV/EHU the question has a final statement "Explain the option you choose"

To answer Q1 correctly, students should justify the difference between the current and potential difference in the open circuit. The results are shown in table 1 .

The vast majority of students (more than three-fourths) in both UPV/EHU and UW chose the correct option. This could indicate that students had learned a basic declarative or descriptive knowledge that helped them distinguish between current and potential difference in a DC circuit. The results indicate an improvement over other studies showing that secondary students confuse the concepts of current and potential difference [8].

Table 1. Result for question Q1

\begin{tabular}{ccc}
\hline Options & $\begin{array}{c}\text { UPV/EHU-Donostia } \\
(\mathrm{N}=72)\end{array}$ & $\begin{array}{c}\text { UW-Seattle } \\
(\mathrm{N}=214)\end{array}$ \\
\hline Zero & $18 \%$ & $9.5 \%$ \\
$6 \mathrm{~V}$ & $0.0 \%$ & $0.5 \%$ \\
Infinity & $0.0 \%$ & $0.5 \%$ \\
$+12 \mathrm{~V} *$ & $79.0 \%$ & $89.0 \%$ \\
No suf. Info. & $3.0 \%$ & $0.5 \%$ \\
\hline
\end{tabular}

The correct one is shown by *

However, in Q1, UPV/EHU students had not only to select the correct answer but also to justify their answer. In this university, we found that only $23 \%(\mathrm{~N}=17)$ of the students 
who chose the correct answer justified their response with a coherent scientific explanation. They explained that the potential difference between points $\mathrm{A}$ and $\mathrm{B}$ is the same that between the battery poles (if the resistance of the wires is ignored). The majority of these answers ( $\mathrm{N}=14,19.5 \%$ of the total answers) explain explicitly that there will be a transitory current in a very brief time interval. For example: "during a very brief interval of time there will be a transient current. In stationary situation there are 12 volts and in the other $0, I$ consider that there is not resistance in the wire".

The majority of the students (57\%) did not explain why they had chosen their answer. They were not able to give an explanation for what happens in the circuit, although most of them knew what the correct answer was.

The option "zero" was chosen by a significant percentage of students at UPV/EHU (18.0\%). A third of the subjects that chose this option explained that the potential difference is zero because the circuit was open. These answers assume that the relationship between current and potential difference is proportional as in Ohms' law. An example of this kind of answer: "the potential difference is proportional to the current. The circuit is open and the current is zero. So, the potential difference is zero". This mistake is consistent with previous studies that showed High School students tend to confuse the concepts of current and potential difference [9]. Another third of the answers in this option assumed that the potential at point $\mathrm{A}$ is the same as the point $\mathrm{B}$ and so, the potential difference is zero. The rest of the answers were unsupported.

The context of question Q2 does not involve an electric circuit and it is an open-ended question designed to probe students' explanations (figure 2). Question Q2 was designed to address the explanatory model that students use for explaining the transitory movement of charges in the system. The aim of this question is to inquire about the students' use of concepts like electric potential and charge quantity in their explanatory model.

Question Q2.- Two conducting spheres of different radii are connected to two batteries as shown in the figure below. If both switches are closed simultaneously, will be electrical current? Will be the spheres become charged? If so, calculate the charge on each. Explain your answers.

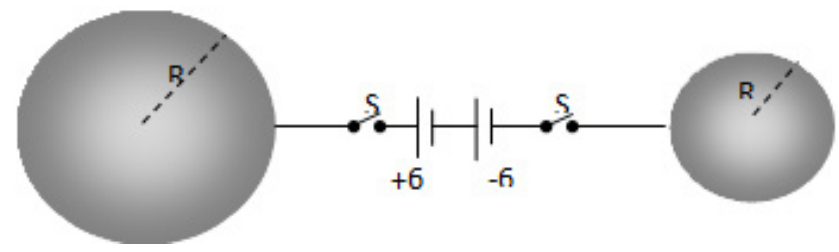

Figure 2. The system consists of a battery, wires and two conducting spheres and is similar to the system in question 1 , but here the context is more relevant to the process of charging bodies (electrostatic) than the context of DC circuits (electrodynamic).

The students were asked if the spheres will charge or not, and if they do become charged, students were also asked to explain why and calculate the charge of each sphere at the end of the process. In question Q2, students were told that the wires were sufficiently long such that there are no interactions (electric induction) between spheres, and the spheres and the battery poles. The two batteries are connected in series, in a way that the big sphere is linked to the $+6 \mathrm{~V}$ pole and the small sphere is linked to the $-6 \mathrm{~V}$.

One way of answering this question correctly, the students should relate the potential difference to the charging process of the spheres, and understand that the charging process will finish when the potential difference between the battery and the two spheres is zero (category A.1 in table 2).

Drude's model, which is the one students have learned, states that to move charges between two points of a conducting wire, a potential difference must exist between two points on the wire. In a correct explanation of question Q2, students have to understand the theoretical model that relates the movement of charges to the amount of potential difference, and they have to be able to use the equation which relates the potential with the charge, in order to calculate the charge of the sphere at the end of the process. If students had been told about surface charge distributions in simple circuit wires, the question could be explained using the mechanism of surface charge gradients and argued by electric field superposition [10]. But this is not the case.

Table 2. Categories of explanation for question Q2

\begin{tabular}{lcc}
\hline \multicolumn{1}{c}{ Category of explanation } & $\begin{array}{c}\text { UPV/EHU-Donostia } \\
(\mathrm{N}=200)\end{array}$ & $\begin{array}{c}\text { UW-Seattle } \\
(\mathrm{N}=504)\end{array}$ \\
\hline $\begin{array}{l}\text { A. }{ }^{*} \text { - The transitory movement of charges is due to a potential difference and the final } \\
\text { quantity of charges is calculated through the Potential and /or Capacitance concepts. }\end{array}$ & $38 \%$ & $42.5 \%$ \\
$\begin{array}{l}\text { A.2 }{ }^{*} \text { - The transitory movement of charges is not mentioned, and the final quantity of } \\
\text { charges is calculated through the Potential and/or Capacitance concepts. }\end{array}$ & $12 \%$ & $0 \%$ \\
B.- They do not become charged. It is not a closed circuit. & $20 \%$ & $34 \%$ \\
C.- Answers based on incoherently justified or not justified statements & $22 \%$ & $17 \%$ \\
D.- Not answered & $8 \%$ & $6.5 \%$ \\
\hline
\end{tabular}

The correct one is shown by * 
Another way of answering correctly the question is to consider the final equilibrium situation without mentioning the electric current. In this answer it is reasoned that the equilibrium is reached when the big sphere has a $+6 \mathrm{~V}$ potential, and the small sphere has a $-6 \mathrm{~V}$ potential. Later, the charge quantity of each sphere is calculated by using the capacitance. Only $12 \%$ of students at UPV/EHU and no one at UW, answer in this way (category A.2 in table 2). This could be due to the fact that the question was asked after studying DC circuits and electrostatics was far from their close experience in learning, or that they don't connect concepts from electrostatic with electric current.

As our current study differs from others in that we focus on students' understanding of the physical justification of the explanatory model for the DC circuits, in table 2 we show not only the correct or incorrect answer, but also different students' categories of explanations.

At both universities, the two main explanatory tendencies were the same (categories A and B).

In category A.1 (about 40\%) are grouped those responses that account for the movement of charges based on a potential difference between the ends of the conductor. This type of response can be interpreted as an explanation based on the Drude's model. The vast majority of answers follows a linear causal reasoning [11] that has a chain of cause and effect: a) There is current because there is a potential difference between two points of the conductor, b) at the end of the process the potential in the two spheres will be the same, as the current ceases, c) applying the potential equation of a sphere, the concept of charge and potential are related, and the final charge on each sphere can be calculated. Standard examples of this category of response are:

"There will be current until the potential difference will be equal between the spheres and the poles of the battery. The final potential is equal in both spheres, but they have opposite signs. The charge per unit area is: $Q_{I}=q_{1} / 4 \pi R_{I}^{2}$ and $Q_{2}=q_{2} / 4 \pi R_{2}{ }^{2}$. When we apply the equation $V=q / \pi \varepsilon r$ which is the potential of a sphere and we take that the potential is the same in both spheres but opposite in sign, we can calculate the charge of each of the spheres."

"They will become charged and there will be current until spheres potential became: $V_{1}=6 \mathrm{~V}$ and $V_{2}=-6 \mathrm{~V}$. As $C=q / \Delta V$, the calculi of charge is: $q=C V=4 \pi \varepsilon_{0} r V$ "

At the UPV/EHU, category A.2 emerged with $12 \%$ of answers $(16 \%)$, which did not occur at UW. In this category the students' reasoning is related only with electrostatic concepts without reference to transitory current that happens before arriving to equilibrium situation. One standard example of this category is:

"Both spheres will have the same final potential, but with opposite signs. The sphere on the left side will have $6 \mathrm{~V}$ and the sphere on the right side will have $-6 \mathrm{~V}$. Applying the definition of the capacitance for a sphere: $C=4 \pi \varepsilon_{0}, r$ and the relation $C=q / V$, then solving for the charge, we obtain the final charge of each sphere: $q=4 \pi \varepsilon_{0} r V^{\prime \prime}$
The majority of the answers (about $70 \%$ at both universities) miscalculates the value of potential and final charge. This failure could indicate students' low level of application of electrostatic equations in the context of transitory currents.

About $20 \%$ of the answers explain that the spheres do not become charged because the circuit is open (category B). Most of them mentioned Kirchhoff's Law to justify the answer. The responses follow a simple causal reasoning [11] that relates one cause with one effect: a) the circuit is open (cause); b) there is no flow of charges (effect). It is clear that these students do not recognize the transient current in this context; they only imagine closed circuit in steady state current. One example of this kind of answer is the following: "The + and - sides of the battery are not connected, so the circuit is not complete. The sum of the potential differences in the circuit is zero"

In category $\mathrm{C}$ we included answers without internal logic or that apply physics concepts without meaning. This type of answers shows that there is not an explicative model to interpret the phenomenon given in question Q2.

\section{Discussion and Implications for Future Work}

When drawing conclusions it is necessary to bear in mind that this study is the initial part of a project on students' explanatory model of DC circuits in both transitory and steady state. The two questions presented describe situations in which current flow is associated with a transitory state. The two questions do not provide conclusive evidence on all university students' explanatory model about transitory state current processes, but the data show that the explanatory categories manifest themselves in two different universities (and countries) and provide further evidence of their persistence. Some of the difficulties we identify are news; others may be interpreted as previously documented difficulties that tend to arise in the first year at university [4].

We found that there are two major explanatory tendencies of current in transitory states. One category explains the movement of charges qualitatively according to the potential difference but there are fewer responses in this category when the explanation is quantitative. Namely, in Q2 about $40 \%$ of the students were able to explain it qualitatively, but only about $23 \%$ of them gave a quantitative explanation as well. Explanations of students in this category are usually accompanied by a linear causal reasoning that associates electric current between two points with the potential difference between them. This explanatory model can be regarded as an application of the Drude's model, which is explained in the instruction of the students involved in this research. A minority of answers from UPV/EHU (12\%) calculate correctly the final charge of the spheres in question Q2 but without mention to the electric current. For this minority of answers, all we can say is that students didn't discuss the transient-state of current. 
The second explanatory category ( $20 \%$ in Q1, $20-34 \%$ in Q2) does not take into account the transient motion of charges in circuits. The explanation is associated with a simple causal reasoning which links the "open circuit" to the fact that there cannot be an electric current in steady states. This line of reasoning is related to a supposed dichotomy, that is, there are only two options: a) open circuit, no current; b) closed circuit, steady-state current. It seems that students in this category have only one explanatory model for a steady-state current.

The results obtained show that a significant percentage of students cannot properly interpret simple transitory state current phenomena. This suggests several possibilities for instructional changes. According to our findings and the explanatory model of current in a wire, the key idea that will be necessary to emphasize would be the microscopic mechanism of production of potential difference between two points within the circuit wire, in both transitory current and steady situations. In particular, this involves the relationship between the concept of electric potential studied in Electrostatics (capacitors, charging bodies ...) with those analyzed in DC circuits. The results show that omitting an explanation of a microscopic mechanism of charge movement makes it harder for students to interpret transitory states in DC circuits. Part of our work will involve the development of a guided problem-solving (GPS) tutorial to help students better understand DC circuits and the connection between the fundamental concepts of electricity (field, potential difference) and an explanatory model of DC circuits.

The present study is the beginning of a project about students' model for explaining electric current in transitory and stationary situation. Regarding previous studies, this study provides new information about the students' ideas/models for explaining the electric current in transitory situations, but it will be necessary more research to confirm and develop the results obtained here, and to extend the information to electrical current in stationary situation in DC circuits. This will be the aim of our next studies.

\section{Acknowledgements}

This work is supported by the project granted by the Government of the Basque Country (Spain). We would like to thank the members of the of the Physics Education
Research Group at the University of Washington, and our colleague Paula Heron for her continued collaboration on this work.

\section{REFERENCES}

[1] D. J. Griffiths (1999). Introduction to Electrodynamics (Prentice-Hall, 1999) Chapter 7, p. 289.

[2] M.A. Heald, Electric field and charges in elementary circuits, Am. J. Phys, 52, 522 (1984)

[3] D. P. Smith and P. van Kampen, Teaching electric circuits with multiple batteries: A qualitative approach. Phys. Rev. ST Phys. Educ. Res 7, 020115 (2011)

[4] J. Guisasola, Teaching and Learning Electricity: The Relations between Macroscopic Level Observations and Microscopic Level Theories. In International Handbook of Research in History, Philosophy and Science Teaching, edited by M.R. Matthews (Springer, Dordrecht, 2014) vol I, chapter 5, 129-156.

[5] K. Zuza, J.M.Almudí, A. Leniz, and J. Guisasola, “Addressing students' difficulties with Faraday's law: A guided problem solving approach", Phys. Rev. ST Phys. Educ. Res 10, 010122 (2014)

[6] L. C. McDermott, P. S. Shaffer, and the P.E.G. at the U.W., Tutorials in Introductory Physics (Pearson Learning Solutions, 2011)

[7] M. Watts, G. Gould, and S. Alsop, "Questions of understanding: Categorising pupils' questions in Science” Social Science Research, 79, 57 (1997)

[8] J. Dupin and S. Joshua, Conceptions of French pupils concerning electric circuits: Structure and evaluation. Journal of Research in Science Teaching, 6, 791 (1987)

[9] L.Liegeois and E. Mullet, High School Students' Understanding of Resistance in Simple Series Electric Circuits, International Journal of Science Education, 24(6), 551 (2002).

[10] R. Chabay and B. Sherwood, Matters and Interactions vol 2. John Willey \& Sons, 3th edition, (2011).

[11] F. Halbwachs, Reflexions sur la causalité physique (reflections on causality in physics), in Les Théories de la Causalité, edited by M. Bunge, F. Halbwachs, T.S. Kuhn, J. Piaget, and L. Rosenfeld (Presses Universitaires de France, Paris, 1971), pp. 19-38 and pp. 39-111. 